\title{
Author's Toolkit
}

\author{
Writing Better Technical Papers \\ Ron Read \\ Human Global Communications Co., Ltd. \\ (formerly Kurdyla and Associates)
}

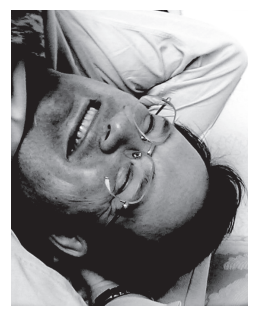

One of the most basic types of explanation in a technical paper is giving the reason for an action or state. Naturally, this should be done clearly.

\section{Reasonable Reasons}

A previous column covered typical problems in expressing cause and effect. Here, we take up additional issues in this important task.

Within a sentence, the most common word used to introduce a cause for something is "because," which joins two clauses:

$\bigcirc$ The robot has extra shielding on its joints because it operates many hours outside.

O Because the robot operates many hours outside, it has extra shielding on its joints.

When a clause is connected to a noun phrase as the reason, "because" is followed by "of":

$\bigcirc$ Extra shielding is needed at the joints because of the harsh weather conditions.

However, this can seem awkward when sentences already use phrases containing "of":

? Most of the transitions of temperature are because of structural instability.

In such cases, a useful alternative is "due to":

Most of the temperature transitions are due to structural instability.

Remember that the word "cause" can be flexibly used as both a noun and a verb:

$O$ This excessive fluctuation in current load is the cause of the accelerated power drain.

$O$ This excessive fluctuation in current load causes the accelerated power drain.

Various other words can be used to express causation:

$\bigcirc$ This excessive fluctuation in current load accounts for the accelerated power drain.

$\bigcirc$ The accelerated power drain is attributed to this excessive fluctuation in current load.

Another approach is to use a more direct verb of action that clearly implies a cause:

$\bigcirc$ This excessive fluctuation in current load accelerates the power drain.
Sometimes the author's meaning becomes confusing when more than one "cause link" appears in a single sentence:

x Signals are sometimes misdirected because the proper transmission path cannot be clearly determined due to local topography.

The above sentence can be understood, but it may require two or three re-readings (readerunfriendly). It's thus better to distinguish the links of causation:

O Signals are sometimes misdirected because the proper transmission path cannot be clearly determined, which is due to variations in the local topography.

Signals are sometimes misdirected, since the local topography can make it difficult to clearly determine the proper transmission path.

Finally, I frequently observe the lack of an explicit indication of cause:

$x$ The survey responses did not vary with the color of the stimulus. We ruled out color as a significant factor.

The survey responses did not vary with the color of the stimulus. Therefore, we ruled out color as a significant factor.

Although the first example is basically clear, the easy addition of "therefore" is a powerful way to help your reader by guiding his/her thinking.

\section{Acknowledgment? Acknowledgments?}

Although a few journals may always use one or the other, the simplest solution is to use the singular when you're thanking only one person or funding source, the plural when thanking two or more people or funding sources.

GrammarQuestions?read@athuman.com

\section{Mini Quiz: What's Wrong?}

1) This is a basic principal of genome theory.

2) The principle cause of this reaction is...

3) In principle, any medium can be used to... (Answers: 1) change to "principle"; 2) change to "principal"; 3) OK) 